\title{
openheart Heart failure following STEMI: a contemporary cohort study of incidence and prognostic factors
}

\author{
Johannes M I H Gho, ${ }^{1}$ Pieter G Postema, ${ }^{2}$ Maartje Conijn, ${ }^{1}$ \\ Nienke Bruinsma, ${ }^{2}$ Jonas S S G de Jong, ${ }^{2,3}$ Connie R Bezzina, ${ }^{2}$ Arthur A M Wilde, ${ }^{2}$ \\ Folkert W Asselbergs ${ }^{1,4,5}$
}

\section{To cite: Gho JMIH, Postema PG, Conijn $\mathrm{M}$, et al. Heart failure following STEMI: a contemporary cohort study of incidence and prognostic factors. Open Heart 2017;4:e000551. doi:10.1136/ openhrt-2016-000551}

JMIHG and PGP shared first authorship.

Received 16 0ctober 2016 Revised 14 May 2017 Accepted 15 August 2017

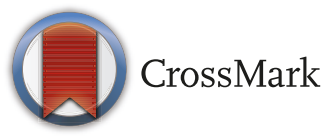

'Department of Cardiology, Division Heart and Lungs, University Medical Center Utrecht, Utrecht, The Netherlands

${ }^{2}$ Department of Clinical and Experimental Cardiology, Heart Center, Academic Medical Center, Amsterdam, The Netherlands

${ }^{3}$ Heart Center, Onze Lieve Vrouwe Gasthuis, Amsterdam, The Netherlands

${ }^{4}$ Durrer Center for Cardiogenetic Research, ICIN-Netherlands Heart Institute, Utrecht, The Netherlands

${ }^{5}$ Institute of Cardiovascular Science, Faculty of Population Health Sciences, University College London, London, UK

Correspondence to Professor Folkert W Asselbergs; f.w.asselbergs@umcutrecht.n|

\section{ABSTRACT}

Objective The aim of the current study was to determine the contemporary incidence, risk factors and prognosis of heart failure (HF) after ST-elevation myocardial infarction (STEMI).

Methods We used the Arrhythmia Genetics in the Netherlands observational cohort study to identify patients with a first STEMI from 2001 onwards $(n=1459)$. HF during follow-up was defined as hospitalisation for HF or an outpatient clinic visit for HF. Cox regression was performed to estimate the relationship between baseline covariates and the onset of HF.

Results Follow-up was completed for 1360 (93.2\%) patients with an overall median follow-up time of 6.7 years, $1232(90.6 \%)$ of these patients had undergone primary percutaneous coronary intervention $(\mathrm{PCl})$. A total of 85 patients $(6.3 \%)$ developed HF during follow-up. HF cases were significantly older at their index $\mathrm{Ml}$ ( 59.9 vs 57.2 years, $\mathrm{P}<0.001$ ) and more commonly had a history of atrial fibrillation $(6.1 \%$ vs $1.4 \%, P=0.001)$ than controls without HF. The crude incidence rate of HF after STEMI was 9.7 (95\% Cl 7.7 to 11.8) per 1000 person-years. In multivariable analysis, peak creatine kinase MB (CK-MB) levels (HR 1.11 per $100 \mathrm{U} / \mathrm{L}(95 \% \mathrm{Cl} 1.11$ to 1.22$)$ ) and a left anterior descending artery (LAD) culprit lesion (HR 2.88 (95\% Cl 1.53 to 5.40$)$ ) were risk factors associated with HF.

Conclusions We found a relatively low long-term contemporary incidence of HF after a first STEMI in the current $\mathrm{PCl}$ era in comparison with other reports. Higher CK-MB levels and a LAD culprit lesion at index STEMI were important risk factors for the development of HF after STEMI.

Trial registration number NCT03007199; Results.

\section{INTRODUCTION}

Heart failure (HF) is a major medical problem in the western world; it is associated with substantial morbidity and mortality and contributes in a significant extent to national healthcare costs. ${ }^{2}$ HF is predominantly caused by the sequelae following ischaemic cardiac events, especially with increasing age. In the current era of primary percutaneous coronary intervention (PCI) for the treatment of ischaemic events (particularly

\section{KEY QUESTIONS}

What is already known about this subject? Following myocardial infarction, patients are at increased risk for heart failure (HF), especially with increasing age.

What does this study add?

Using a contemporary patient cohort recruited in the Netherlands with a first ST-elevation myocardial infarction (STEMI), we observed that $6.3 \%$ of patients developed HF during a median follow-up of 6.7 years. The median time to onset of HF was 2.1 years. Higher CK-MB levels and a leftanterior descending artery culprit lesion at index STEMI were important risk factors for the development of HF after STEMI.

How might this impact on clinical practice? These findings should encourage clinicians to closely monitor patients with important risk factors for $\mathrm{HF}$ following STEMI.

for ST-elevation myocardial infarction or STEMI), it could be that the incidence of $\mathrm{HF}$ is influenced by an increased survival following the ischaemic event due to rapid and successful PCI and because of demographic changes, for example, ageing population. ${ }^{1}$ Data on the long-term incidence of HF after STEMI in the current PCI era is scarce and also historically varies among different studies. For example, in a recent nationwide Swedish registry study (SWEDEHEART), HF incidence during index hospitalisation declined from $50 \%$ to $28 \%$ in the subgroup including STEMI patients between 1996 and 2008. Among others, this might indeed partly reflect the changes that occurred in the reperfusion treatment during the study period (from predominantly thrombolysis to predominantly primary PCI). ${ }^{3}$ In comparison, in a Canadian cohort study, $13.6 \%$ of the STEMI patients were diagnosed with HF during index myocardial infarction (MI) hospitalisation with a 1-year cumulative HF 
incidence of $23.4 \%$ between 2002 and 2008. ${ }^{4}$ In the same study, development of HF was associated with a 1-year mortality of $9.9 \%$ in the subset of STEMI patients. ${ }^{4}$ Here, we aimed to determine the contemporary incidence and prognostic factors associated with HF after STEMI in the current PCI era.

\section{METHODS}

\section{Study design}

The Arrhythmia Genetics in the NEtherlandS (AGNES) study is a Dutch multicentre observational study of which details have been published before..$^{5-7}$ The AGNES cohort consisted of individuals with a first STEMI. This manuscript has been published in part as a PhD thesis. ${ }^{8}$

\section{Setting and participants}

The investigation conforms with the principles outlined in the Declaration of Helsinki. ${ }^{9}$ Dutch patients of European descent with first STEMI were recruited at seven heart centres in The Netherlands from 2001 to 2013. In the AGNES study, patients with ECG registered ventricular fibrillation (VF) or without VF occurring before reperfusion therapy were included. Excluded were individuals with an actual non-ST-elevation myocardial infarction (NSTEMI), prior MI or revascularisation prior to inclusion, congenital heart defects, known structural heart disease, severe comorbidity at discretion of the operator that would result in severely decreased prognosis, severe electrolyte disturbances, trauma at presentation, recent surgery, previous coronary artery bypass graft or use of class I and III antiarrhythmic drugs. Patients who died within 30 days of the index infarct, and patients with an incomplete follow-up were excluded from this analysis.

\section{Data sources}

Data on age, gender, cardiovascular risk factors, medication use before STEMI, infarct characteristics (eg, type of reperfusion therapy, biomarkers and culprit lesion) were recorded at admission for index MI. Data on risk factors and family history (FH) in a subcohort were previously defined. ${ }^{7} \mathrm{FH}$ included sudden death (SD) and cardiovascular disease (CVD). A positive $\mathrm{FH}$ of SD was defined as a self-reported parent or sibling who died suddenly and unexpectedly. We collected follow-up data from the hospital of index MI admission and the referral centre. Collected endpoints included subsequent MI, stroke, HF, revascularisation (coronaryartery bypass grafting (CABG) and PCI), implantable cardioverter-defibrillator (ICD) implantation, aborted cardiac arrest and all-cause mortality. Hence, information on the medical status of every patient included in the study was collected. In case of no events in the years after the first MI, no endpoints were scored, and only the correspondence regarding the last outpatient clinic visit was obtained. In case of reached endpoints, a form was filled in proposing the endpoint, data of endpoint and necessary remarks. Also, all available documents regarding these endpoints were collected. These included clinical letters, ECGs and correspondence regarding the last visit to the outpatient clinic. The forms and documents were reviewed by the adjudication committee (PGP and FWA), who verified the endpoints based on the available information. If patients were seen in another hospital, records were requested for follow-up. In case of insufficient data, we contacted general practitioners for additional information. In this study, we defined cases with HF as patients with hospital admission for $\mathrm{HF}$ or patients visiting the outpatient clinic for HF during follow-up. Patients without HF during follow-up served as control cases. Mortality data were verified using the Dutch Municipal Personal Records Database.

\section{Statistical methods}

Baseline patient characteristics, index MI characteristics and outcomes are described using descriptive statistics. Differences in continuous variables between cases with HF and controls without HF were tested using an independent t-test when data were normally distributed or with a Mann-Whitney test when no normal distribution was present. Differences in categorical variables between cases and controls were determined using a $\chi^{2}$ test. We calculated cumulative incidence (cumulative percentage) and incidence rates (cases per 1000 person-years) of HF. Kaplan-Meier curves were generated to describe unadjusted cumulative HF incidence and all-cause mortality in STEMI patients using 5-year follow-up.

The associations of exposures of interest with the onset of HF were explored using Cox proportional hazard models. HF was the primary outcome measure, either determined by hospital admission for HF or visiting the outpatient clinic for HF. Cox regression with complete case analysis was performed to estimate the relationship between baseline prognostic factors and the development of HF after MI and to determine HR with $95 \%$ CI. The proportional hazards assumption was investigated by partial residual plots. In all analyses, a $\mathrm{P}$ value of $<0.05$ was considered as significant. Statistics were performed using IBM SPSS Statistics (V.22) and R (V.3.2.3).

\section{RESULTS}

In the AGNES cohort $(n=1459)$, follow-up was incomplete for 74 patients due to insufficient available information (inability to retrieve follow-up information) and 25 patients died within 30 days of index MI. Follow-up was thus completed for 1360 patients. In total, 85 patients developed HF during follow-up (cases) and 1275 did not develop HF during follow-up (controls) (figure 1).

\section{Baseline and infarct characteristics}

Cases with HF were significantly older at their index MI ( 59.9 vs 57.2 years, $\mathrm{P}<0.001$ ), more commonly had a positive $\mathrm{FH}$ of $\mathrm{CVD}(77.5 \%$ vs $65.8 \%, \mathrm{P}=0.032)$, a positive $\mathrm{FH}$ of SD ( $41 \%$ vs $26.1 \%, \mathrm{P}=0.006)$, a history of atrial fibrillation $(6.1 \%$ vs $1.4 \%, \mathrm{P}=0.001)$ and were less likely to use statins prior to MI compared with controls (3.6\% vs $10.6 \%$, $\mathrm{P}=0.041$ ) (table 1). In patients with HF, median peak 


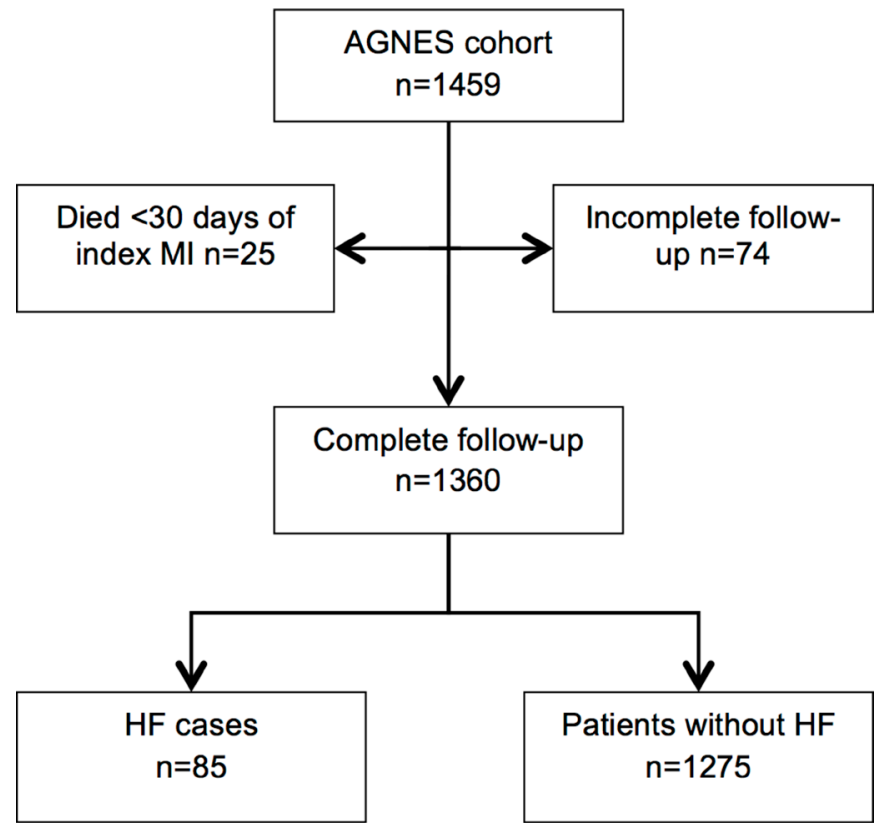

Figure 1 Flow chart. AGNES, Arrhythmia Genetics in the NEtherlandS; HF, heart failure; MI, myocardial infarction.

CK-MB levels at index STEMI were significantly higher (422.5 U/L, IQR 333.0) than in controls (181.0 U/L, IQR 259.0) $(\mathrm{P}<0.001) \quad($ table 2$)$. Patients with HF were more likely to have suffered from a leftanterior descending artery (LAD) as culprit lesion (82.3\% vs $49.8 \%, \mathrm{P}<0.001)$, whereas controls were more likely to have an RCX $(3.8 \%$ vs $15.9 \%, \mathrm{P}=0.003)$ or RCA as culprit lesion $(12.7 \%$ vs $33.3 \%, \mathrm{P}=0.001)$ at index STEMI. Other baseline characteristics were comparable between the two groups. There was no significant difference in $\mathrm{VF}$ at index MI between cases $(48.2 \%)$ and controls $(41.3 \%)$.

\section{HF incidence after MI and survival}

A total of $85(6.3 \%)$ patients developed HF during an overall median follow-up time of 6.7 years (table 3 ). Median follow-up was longer in cases with $\mathrm{HF}$ than in controls (9.4 vs 6.4 years, $\mathrm{P}<0.001)$. The median time until the onset of HF was 2.1 years. We observed 12 $(0.9 \%)$ patients developed HF within 7 days from index STEMI. The crude incidence rate of HF following a first STEMI was 9.7 (95\% CI 7.7 to 11.8 ) per 1000 personyears. In patients 65 years or older, the incidence rate of $\mathrm{HF}$ was 14.1 (95\% CI 8.9 to 19.4) per 1000 personyears, compared with 8.4 (95\% CI 6.2 to 10.6$)$ in patients younger than 65 years. During follow-up, cases with $\mathrm{HF}$ were more likely to have been subjected to revascularisation by additional PCI of the initial culprit artery (14.1\% vs $7.4 \%, \mathrm{P}=0.025)$ or $\mathrm{CABG}(10.6 \%$ vs $5.3 \%$, $\mathrm{P}=0.042)$ than controls. We also found a higher rate of ICD implantation $(44.7 \%$ vs $3.5 \%, \mathrm{P}<0.001)$ and stroke $(7.1 \%$ vs $2.6 \%, \mathrm{P}=0.017)$ in cases compared with controls.

Table 1 Baseline characteristics

\begin{tabular}{|c|c|c|c|c|}
\hline & With HF $(n=85)$ & Without HF $(n=1275)$ & $\begin{array}{l}\text { Number of } \\
\text { missings }\end{array}$ & $P$ value \\
\hline Gender (female) & $20(23.5)$ & $261(20.5)$ & 0 & 0.500 \\
\hline Mean age in years at index infarction (SD) & $59.9(10.3)$ & $57.2(10.7)$ & 0 & $<0.001$ \\
\hline VF at index Ml & $41(48.2)$ & $527(41.3)$ & 0 & 0.212 \\
\hline \multicolumn{5}{|l|}{ Cardiovascular risk profile } \\
\hline Mean BMI (SD) & $26.6(3.9)$ & $26.6(3.9)$ & 61 & 0.806 \\
\hline FH of CVD & $62(77.5)$ & $817(65.8)$ & 39 & 0.032 \\
\hline Current smoker & $50(59.5)$ & $767(61.5)$ & 29 & 0.718 \\
\hline Diabetes & $8(9.6)$ & $94(7.6)$ & 41 & 0.502 \\
\hline Hypertension & $32(39)$ & $378(31.3)$ & 72 & 0.148 \\
\hline Atrial fibrillation & $5(6.1)$ & $17(1.4)$ & 58 & 0.001 \\
\hline High cholesterol & $17(22.7)$ & $378(32.1)$ & 109 & 0.087 \\
\hline FH of sudden death & $34(41)$ & $330(26.1)$ & 14 & 0.006 \\
\hline Angina 48 hours before STEMI & 37 (45.1) & $455(36.6)$ & 35 & 0.122 \\
\hline \multicolumn{5}{|l|}{ Cardiac medication before STEMI } \\
\hline$\beta$-blocker & $13(15.7)$ & $120(9.6)$ & 24 & 0.073 \\
\hline Statins & $3(3.6)$ & $133(10.6)$ & 24 & 0.041 \\
\hline Diuretics & $5(6.1)$ & $79(6.3)$ & 23 & 0.943 \\
\hline ACE inhibitors/ARB & $11(13.3)$ & $98(7.8)$ & 26 & 0.080 \\
\hline Aspirin/oral anticoagulation & 9 (10.8) & $103(8.2)$ & 22 & 0.401 \\
\hline
\end{tabular}

Numbers in the columns with and without HF denote $\mathrm{n}(\%)$, unless specified otherwise.

ARB, angiotensin receptor blocker; BMI, body mass index; CVD, cardiovascular disease; FH, family history; HF, heart failure;

MI, myocardial infarction; STEMI, ST-elevation myocardial infarction; VF, ventricular fibrillation. 
Table 2 Characteristics of index STEMI

\begin{tabular}{|c|c|c|c|c|}
\hline & With HF $(n=85)$ & Without HF $(n=1275)$ & $\begin{array}{l}\text { Number of } \\
\text { missings }\end{array}$ & $P$ value \\
\hline Reperfusion therapy & & & 10 & \\
\hline $\mathrm{PCl}$ & $73(85.9)$ & $1159(90.9)$ & & 0.125 \\
\hline CABG & $3(3.5)$ & $19(1.5)$ & & 0.149 \\
\hline Thrombolysis & $4(4.7)$ & $34(2.7)$ & & 0.269 \\
\hline $\begin{array}{l}\text { None (spontaneous reperfusion on angiography or } \\
\text { medication) }\end{array}$ & $5(5.9)$ & $53(4.2)$ & & 0.446 \\
\hline Time between symptoms and $\mathrm{PCI}(\mathrm{min})$ & 180 (IQR 112) & 180 (IQR 119) & 0 & 0.670 \\
\hline Maximal troponin $\mathrm{T}$ value (median) $\mu \mathrm{g} / \mathrm{L}$ & $6.26($ IQR 8.74$)$ & 2.10 (IQR 4.03) & 1050 & 0.280 \\
\hline Maximal CK-MB value (median) U/L & 422.5 (IQR 333.0) & 181.0 (IQR 259.0) & 243 & $<0.001$ \\
\hline Culprit lesion & & & 55 & \\
\hline $\mathrm{LAD}$ & $65(82.3)$ & $610(49.8)$ & & $<0.001$ \\
\hline $\mathrm{RCX}$ & $3(3.8)$ & $195(15.9)$ & & 0.003 \\
\hline $\mathrm{RCA}$ & $10(12.7)$ & 409 (33.3) & & $<0.001$ \\
\hline LM & $1(1.3)$ & $12(1.0)$ & & 0.829 \\
\hline Multivessel disease & $30(38.5)$ & $450(36.9)$ & 62 & 0.780 \\
\hline
\end{tabular}

Numbers in the columns with and without HF denote $n(\%)$, unless specified otherwise.

CABG, coronary artery bypass grafting; HF, heartfailure; LAD, left anterior descending artery; LM, left main artery; PCI, percutaneous coronary intervention; RCA, right coronary artery; RCX, ramus circumflexus; STEMI, ST-elevation myocardial infarction.

Overall $4.4 \%$ of the patients developed HF during 5 -year follow-up (figure 2). During follow-up 23.5\% of cases with $\mathrm{HF}$ versus $11.1 \%$ controls died $(\mathrm{P}=0.001)$. Overall 5 -year mortality after a first STEMI was $6.7 \%$ (figure 3 ).

\section{Risk factors associated with the onset of HF following index STEMI}

The proportional hazards assumption was violated, and therefore the dataset was administratively censored at 10-year follow-up to ensure goodness-of-fit. A multivariable complete case analysis $(\mathrm{n}=834)$ for the outcome of HF was performed using age at index STEMI, gender, peak CK-MB levels, the LAD culprit lesion and the calendar year of the index STEMI as predictors. Predictors associated with HF were peak CK-MB levels (HR 1.11 per $100 \mathrm{U} / \mathrm{L}$ (95\% CI 1.11 to 1.22)), a LAD culprit lesion (HR 2.88 (95\% CI 1.53 to 5.40)) and the year of index STEMI (HR 0.89 per year (95\% CI 0.83 to 0.96$)$ ). Furthermore, we found a trend towards an association with higher age at STEMI (HR 1.02 (95\% CI 1.00 to 1.05)). We did not find an association of gender with the outcome HF (male HR 0.78 (95\% CI 0.41 to 1.50$)$ ).

\section{DISCUSSION}

Using a patient cohort recruited in the Netherlands with a first STEMI, we observed that $6.3 \%$ of patients developed HF during a median follow-up of 6.7 years. The

\begin{tabular}{|c|c|c|c|c|}
\hline & Total $(n=1360)$ & With HF $(n=85)$ & Without HF (n=1275) & $P$ value \\
\hline Median follow-up in years (IQR) & $6.7(2.5-10.9)$ & $9.4(6.5-12.6)$ & $6.4(0.2-10.8)$ & $<0.001$ \\
\hline \multicolumn{5}{|l|}{ Revascularisations } \\
\hline PCI culprit & $106(7.8)$ & $12(14.1)$ & $94(7.4)$ & 0.025 \\
\hline PCI non-culprit & $117(8.6)$ & $10(11.8)$ & $107(8.4)$ & 0.283 \\
\hline CABG & $77(5.7)$ & $9(10.6)$ & $68(5.3)$ & 0.042 \\
\hline ICD & $83(6.1)$ & $38(44.7)$ & $45(3.5)$ & $<0.001$ \\
\hline Second MI & $130(9.6)$ & $10(11.8)$ & $120(9.4)$ & 0.475 \\
\hline Stroke & $39(2.9)$ & $6(7.1)$ & $33(2.6)$ & 0.017 \\
\hline Deaths & $162(11.9)$ & $20(23.5)$ & $142(11.1)$ & 0.001 \\
\hline
\end{tabular}

Numbers in the columns with and without HF denote $n(\%)$, unless specified otherwise.

The $P$ value is derived from the comparison between cases with HF and controls.

CABG, coronary artery bypass grafting; HF, heart failure; ICD, implantable cardioverter-defibrillator; MI, myocardial infarction; PCI, percutaneous coronary intervention; STEMI, ST-elevation myocardial infarction. 


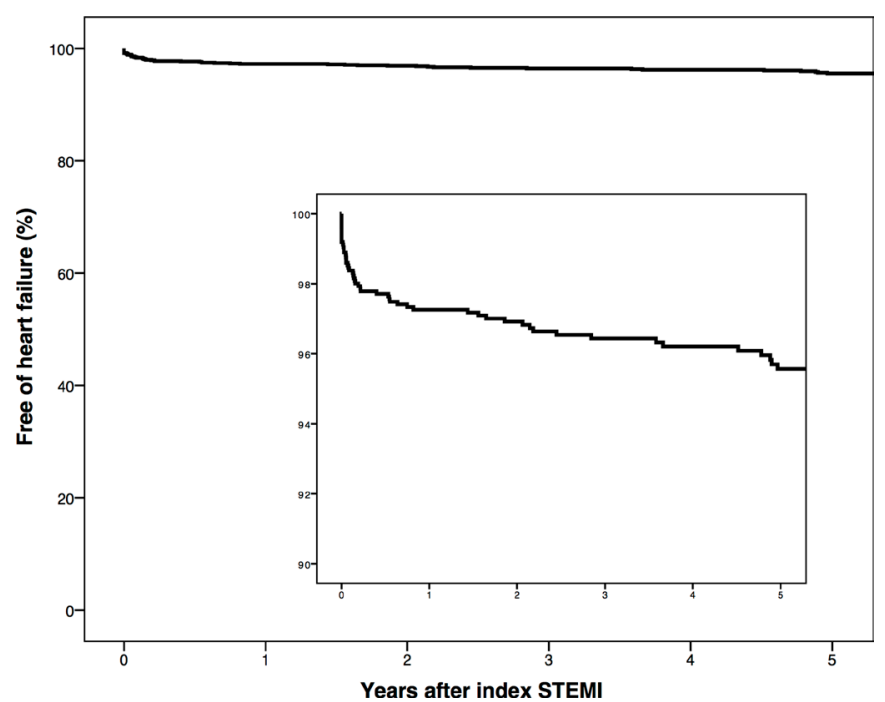

Figure 2 Kaplan-Meier curve showing the time until onset of heart failure in 5-year follow-up. The inset shows the portion of the graph with $100 \%-90 \%$ free of heart failure. STEMI, ST-elevation myocardial infarction.

median time to onset of HF was 2.1 years. The overall mortality was twice as high in the HF group versus the control group after index MI. Higher CK-MB levels and a LAD culprit lesion at index STEMI were important risk factors for the outcome of HF.

\section{Incidence}

Several previous studies have reported the contemporary long-term HF incidence after MI (table 4), and in STEMI subgroups, they showed an HF incidence between $4 \%$ and 28\%. ${ }^{3410}$ When comparing different studies, variability in reperfusion therapy over the years and $\mathrm{HF}$ definition should be taken into account. In the current study, overall $90.6 \%$ of patients underwent primary PCI and 2.8\% thrombolysis, which resembles a unique

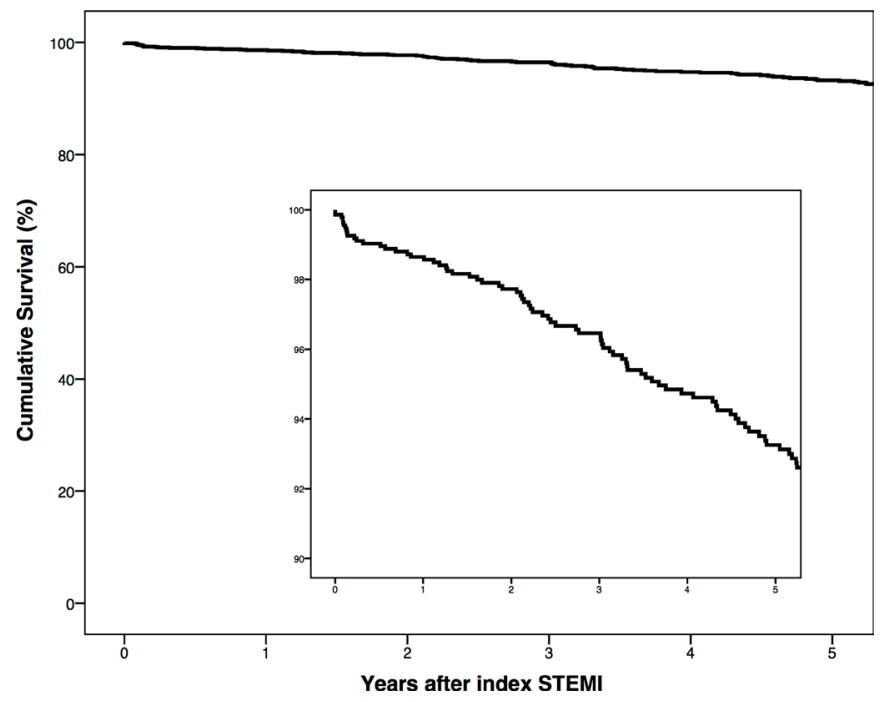

Figure 3 Kaplan-Meier curve showing the overall unadjusted 5-year survival after a first STEMI. The inset shows the portion of the graph with 100\%-90\% cumulative survival. STEMI, ST-elevation myocardial infarction. contemporary STEMI cohort and comparable population studies undergoing similar reperfusion therapy are lacking. Of the SWEDEHEART study, patients with STEMI or left bundle branch block admitted in 20022003, 72.2\% underwent thrombolysis and $2.78 \%$ primary PCI compared with $8.5 \%$ thrombolysis and $91.5 \%$ primary PCI in 2008. ${ }^{3}$ In the Canadian study subgroup of STEMI patients, PCI was performed in $46.7 \%$ of patients with HF during index hospitalisation, in $52.5 \%$ of patients who developed HF after discharge and in $60.2 \%$ of those who did not develop HF. ${ }^{4}$ In a Japanese study enrolling 5429 STEMI patients from 2005 to 2007, HF hospitalisation incidence was $4.4 \%$ per year during the first year after the index STEMI and approximately $1.0 \%$ per year beyond 1 year to 5 years (median follow-up 1956 days). ${ }^{10}$

Other studies were not limited to STEMI but also included patients with NSTEMI and were predominantly from the thrombolytic era. In the Framingham Heart Study, Velagaleti et $a l^{11}$ found that $14.8 \%(21 / 142)$ of patients surviving 30 days after index MI developed congestive HF during 5-year follow-up from 1990 to 1999. In a different Canadian study between 1994 and 2000, among patients with MI 65 years or older without HF during their index hospitalisation, 71\% (3040/4291) developed HF by 5 years. ${ }^{12}$ Data of Torabi $e t$ al showed an HF incidence of 33\% after discharge from hospital admission for index MI. ${ }^{13}$ In a study by Najafi et al, $22.4 \%$ developed HF within 28 days of index admission (after exclusion of patients who died within 28 days), and from these patients, $12.4 \%$ had at least one subsequent admission with HF after 10-year follow-up. ${ }^{14}$ Epidemiological research in Olmsted County (Minnesota, USA) found an $\mathrm{HF}$ incidence of $41 \%$ during a median follow-up of 6.6 years after MI. ${ }^{15}$ In a Norwegian electronic record study (2001-2009), of 69372 hospitalised patients with MI, $17.1 \%$ developed in-hospital HF and another $5.4 \%$ developed postdischarge HF within 1year. ${ }^{16}$ A more recent Danish study found a 90 -day HF incidence of $19.6 \%$ in 2009-2010. ${ }^{17}$

\section{Prognostic factors}

Infarct size and anterior MI are well-known risk factors for adverse prognosis in STEMI patients. ${ }^{18}$ In this study, maximal CK-MB value as a proxy for infarct size was an adverse prognostic factor for the development of HF. Cardiac biomarkers, including peak CK-MB, significantly correlated in previous research with infarct size and left ventricular ejection fraction. ${ }^{19}$ We also found a LAD culprit lesion to be a risk factor for the development of HF after STEMI (HR 2.82). Our study demonstrated a negative association between the year in which the index event occurred and the development of HF. This is in accordance to other studies where decreasing trends in the development of HF after MI are reported..$^{15} 172021$ We also found a trend for higher age at the time of the index MI associated with the development of HF after a first STEMI. This was expected, as age is a well-known risk factor for HF development. ${ }^{3141520}$ For example, Desta 


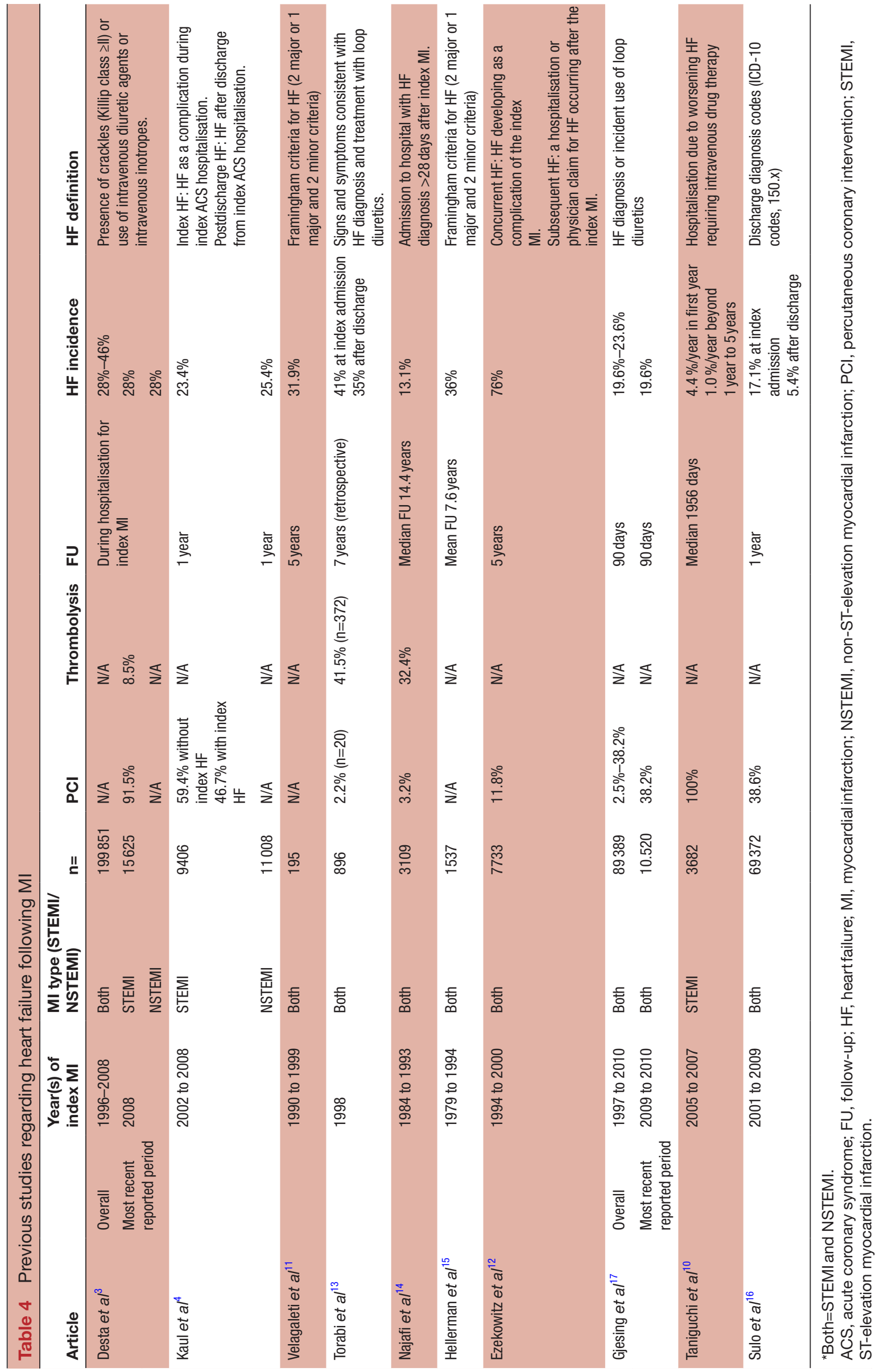

$\frac{0}{8}$ 
et $a l^{\circledR}$ found an OR of 1.05 (95\% CI 1.05 to 1.06 ) for every additional year increase in age. A previous study found a significant association of gender with HF incidence after MI, but we could not replicate this finding in our STEMI cohort. ${ }^{20}$ Regarding medication, a previous Canadian study found that users of beta-blockers and statins after index MI were less likely to develop HF, which would be interesting for future study. ${ }^{12}$

\section{Survival}

We found that about one in four patients with HF died during a median follow-up of 6.7 years. This is consistent with results reported in other studies in which the poor prognosis after onset of HF is described. ${ }^{14152122}$ In the SWEDEHEART study, Desta et al found a decrease in 1-year mortality for patients with clinical HF following MI from $36 \%$ to $31 \%$ between 1996 and $2008 .{ }^{3}$ Kaul et at $t^{4}$ found a 1-year mortality rate of $10.6 \%$ in patients with $\mathrm{HF}$ and $2.4 \%$ mortality in patients without HF post-STEMI.

\section{Limitations}

Cardiovascular endpoints might have been missed as follow-up was performed in the hospital of the index admission (information bias), for example (predominantly milder) cases with HF managed by the general practitioner or cases with HF managed in other hospitals. To reduce information bias, an adjudication committee reviewed the available forms and documents concerning endpoints, and in case of insufficient data, we contacted general practitioners for additional information. In this study, we defined $\mathrm{HF}$ as a hospitalisation or visiting the outpatient clinic for HF, which is of relevance to external validity. Other studies also used administration of diuretics or symptoms of HF to define HF. ${ }^{3} 1317 \mathrm{We}$ were not able to differentiate between $\mathrm{HF}$ with preserved ejection fraction and HF with reduced ejection fraction phenotypes, as we did not have access to detailed imaging parameters in all patients, although it is likely that the majority of cases with HF developed systolic dysfunction after STEMI. The study did not systematically include consecutive STEMI patients, patients with primary VF were selectively included, patients without primary $\mathrm{VF}$ were randomly included and patients with prior MI were excluded, but the cohort represents a contemporary STEMI population.

\section{CONCLUSION}

This research demonstrates a low contemporary incidence of HF after a first PCI-treated STEMI in the Netherlands in comparison with other reports. Higher CK-MB levels and a LAD culprit lesion at index STEMI were important risk factors for the development of HF after STEMI.

Contributors JMIHG designed the current study, performed data collection and analyses, and drafted and revised the manuscript. PGP verified endpoints in the adjudication committee and revised the manuscript critically for important intellectual content. MC performed data collection and analyses and drafted the manuscript. NB performed data collection and critically reviewed and revised the manuscript. JSSGdJ was involved with data collection and analyses in the primary study and critically reviewed and revised the manuscript. CRB and AW conceived the parent study, monitored data collection and critically reviewed and revised the manuscript. FWA designed the current study, verified endpoints in the adjudication committee and revised the manuscript critically for important intellectual content. He is guarantor. All authors gave final approval of the version to be published.

Funding This work was supported by a Dekker scholarship-Junior Staff Member 2014T001 - Netherlands Heart Foundation to [FWA] and a clinical fellowship to [FWA] from the Netherlands Organization for Health Research and Development (ZonMw grant 90700342) and UCL Hospitals NIHR Biomedical Research Centre [FWA]

\section{Competing interests None declared.}

Ethics approval All patients or their legal representative gave written informed consent for inclusion, and collection of information during follow-up was also approved by the institutional ethics committee.

Provenance and peer review Not commissioned; externally peer reviewed.

Data sharing statement There are no additional data available for this paper.

Open Access This is an Open Access article distributed in accordance with the Creative Commons Attribution Non Commercial (CC BY-NC 4.0) license, which permits others to distribute, remix, adapt, build upon this work non-commercially, and license their derivative works on different terms, provided the original work is properly cited and the use is non-commercial. See: http://creativecommons.org/ licenses/by-nc/4.0/

(c) Article author(s) (or their employer(s) unless otherwise stated in the text of the article) 2017. All rights reserved. No commercial use is permitted unless otherwise expressly granted.

\section{REFERENCES}

1. Mosterd A, Hoes AW. Clinical epidemiology of heart failure. Heart 2007;93:1137-46.

2. Ho JE, Enserro D, Brouwers FP, et al. Predicting heart failure with preserved and reduced ejection fraction: the international collaboration on heart failure subtypes. Circ Heart Fail 2016;9.

3. Desta L, Jernberg T, Löfman I, et al. Incidence, temporal trends, and prognostic impact of heart failure complicating acute myocardial infarction. The SWEDEHEART registry (Swedish Web-System for Enhancement and Development of Evidence-Based Care in Heart Disease Evaluated According to Recommended Therapies): a study of 199,851 patients admitted with index acute myocardial infarctions, 1996 to 2008. JACC Heart Fail 2015;3:234-42.

4. Kaul P, Ezekowitz JA, Armstrong PW, et al. Incidence of heart failure and mortality after acute coronary syndromes. Am Heart $J$ 2013;165:379-85.

5. Bezzina CR, Pazoki R, Bardai A, et al. Genome-wide association study identifies a susceptibility locus at 21q21 for ventricular fibrillation in acute myocardial infarction. Nat Genet 2010;42:688-91.

6. de Jong JS, Marsman RF, Henriques JP, et al. Prognosis among survivors of primary ventricular fibrillation in the percutaneous coronary intervention era. Am Heart J 2009;158:467-72.

7. Dekker LR, Bezzina CR, Henriques JP, et al. Familial sudden death is an important risk factor for primary ventricular fibrillation: a casecontrol study in acute myocardial infarction patients. Circulation 2006;114:1140-5.

8. Gho JM. Opportunities in the failing heart. Utrecht: Utrecht University. 2015.

9. Rickham PP. Human experimentation. Code of ethics of the world medical association. Declaration of helsinki. Br Med J 1964;2:177.

10. Taniguchi T, Shiomi H, Morimoto $\mathrm{T}$, et al. Incidence and prognostic impact of heart failure hospitalization during follow-up after primary percutaneous coronary intervention in st-segment elevation myocardial infarction. Am J Cardiol 2017;119:1729-39.

11. Velagaleti RS, Pencina MJ, Murabito JM, et al. Long-term trends in the incidence of heart failure after myocardial infarction. Circulation 2008;118:2057-62.

12. Ezekowitz JA, Kaul P, Bakal JA, et al. Declining in-hospital mortality and increasing heart failure incidence in elderly patients with first myocardial infarction. J Am Coll Cardiol 2009;53:13-20.

13. Torabi A, Cleland JG, Khan NK, et al. The timing of development and subsequent clinical course of heart failure after a myocardial infarction. Eur Heart J 2008;29:859-70.

14. Najafi F, Dobson AJ, Hobbs M, et al. Late-onset heart failure after myocardial infarction: trends in incidence and survival. Eur J Heart Fail 2008;10:765-71. 
15. Hellermann JP, Jacobsen SJ, Redfield MM, et al. Heart failure after myocardial infarction: clinical presentation and survival. Eur $\mathrm{J}$ Heart Fail 2005;7:119-25.

16. Sulo G, Igland J, Nygård $\mathrm{O}$, et al. Prognostic impact of in-hospital and postdischarge heart failure in patients with acute myocardial infarction: a nationwide analysis using data from the cardiovascular disease in Norway (CVDNOR) project. J Am Heart Assoc 2017;6:e005277.

17. Gjesing A, Gislason GH, Køber L, et al. Nationwide trends in development of heart failure and mortality after first-time myocardial infarction 1997-2010: a Danish cohort study. Eur J Intern Med 2014;25:731-8.

18. Sutton MG, Sharpe N. Left ventricular remodeling after myocardial infarction: pathophysiology and therapy. Circulation 2000;101:2981-8.

19. Chia S, Senatore F, Raffel OC, et al. Utility of cardiac biomarkers in predicting infarct size, left ventricular function, and clinical outcome after primary percutaneous coronary intervention for STsegment elevation myocardial infarction. JACC Cardiovasc Interv 2008;1:415-23.

20. Shafazand M, Rosengren A, Lappas G, et al. Decreasing trends in the incidence of heart failure after acute myocardial infarction from 1993-2004: a study of 175,216 patients with a first acute myocardial infarction in Sweden. Eur J Heart Fail 2011;13:135-41.

21. Chen J, Hsieh AF, Dharmarajan K, et al. National trends in heart failure hospitalization after acute myocardial infarction for Medicare beneficiaries: 1998-2010. Circulation 2013;128:2577-84

22. Spencer FA, Meyer TE, Goldberg RJ, et al. Twenty year trends (1975-1995) in the incidence, in-hospital and long-term death rates associated with heart failure complicating acute myocardial infarction: a community-wide perspective. J Am Coll Cardiol 1999;34:1378-87. 